\title{
Simulation des transferts de contamination par les gaz et les aérosols
}

\author{
D. BOULAUD, A. KEREVER, J. L. ROUYER, \\ C. VAVASSEUR et D. VIGLA (*) \\ (Manuscrit reçu le 15 février 1980)
}

\begin{abstract}
RÉSUMÉ
On dresse dans cet article le bilan des principales techniques utilisées par le Service de Protection Technique (S.P.T./S.T.E.P.) du C.E.A. dans la simulation des transferts de contamination. Ces techniques permettent d'optimiser les conditions de ventilation des installations nucléaires, elles ont lieu in situ ou sur maquette.

Le traçage à l'hélium est une méthode particulièrement pertinente pour évaluer les transferts de contamination sous forme gazeuse et déterminer les débits de ventilation. D'autres applications sont en cours de développement. Les techniques de simulation par aérosols tests servent à étudier les fonctions de transfert d'un système selon des procédures normalisées. Lorsque réside un certain nombre d'incertitudes sur la source de contamination, on simule à la fois le transfert et la génération. La génération se fait à partir de poudre $(\mathrm{ZnS} \ldots)$ ou de solutions (solutions de sels de fluorescéine ou de sodium...). Les mesures s'effectuent sur le résidu sec ou sur les vésicules liquides. La qualité de la simulation dépend du respect d'un certain nombre de contraintes découlant de la théorie des similitudes. Un exemple d'application de ces techniques traite du transfert des aérosols de produits de fission émis lors d'une ébullition.
\end{abstract}

\begin{abstract}
A review is made of the chief techniques used by the laboratory to simulate contamination transfers. These techniques make it possible to optimise ventilation conditions in nuclear installations; they can be applied in situ or on models. 1) Helium tracing is particularly relevant to evaluate gaseous contamination transfers
\end{abstract}

(*) Commissariat à l'Énergie Atomique, Institut de Protection et de Sûreté Nucléaire, Département de Protection, Services Techniques de Protection, Service de Protection Technique, B.P. ${ }^{\circ} 6,92260$ Fontenay-aux-Roses. 
and determine ventilation rates. Other applications are being developed. 2) Simulation by test aerosols is used to study transfer functions in a system according to standardized procedures. When there is a number of uncertainties on the contamination source, both the transfer and production are simulated.

Production is made from powder $(\mathrm{ZnS} \ldots)$ or solutions (fluoresceine or sodium salts, ...); measurements are carried out on the dry residue or on liquid vesicles. The quality of simulation will depend on the respect of a number of constraints resulting from the similitude theory. An example of the application of these techniques considers the transfer of fission product aerosols emitted during ebullition.

\section{INTRODUCTION}

La simulation des transferts de contamination, effectuée in situ ou sur maquette, dans des conditions très proches des conditions réelles, est un moyen d'évaluer expérimentalement l'efficacité des dispositions prévues - confinement et ventilation - pour protéger le personnel ou l'environnement contre les risques de contamination. Elle permet de rechercher quelles sont les dispositions les meilleures et contribue ainsi à une plus grande sûreté des installations.

Ayant accompli plusieurs études de simulation des transferts de contamination par les gaz et aérosols, il nous a semblé utile de dresser un bilan de ces travaux afin de préciser les possibilités et les limites des techniques de simulation utilisées.

\section{1. ÉTUDES ET TECHNIQUES DE SIMULATION}

Ces études, demandées principalement pour le retraitement des combustibles irradiés, avaient généralement pour objectif de minimiser les transferts de contamination par les effluents gazeux sous certaines contraintes de ventilation.

Nous avons été notamment amenés à effectuer des simulations concernant : irradiés;

- les transferts de contamination des piscines de stockage de combustibles

- plusieurs systèmes de ventilation de cisaille d'éléments combustibles;

- la ventilation d'un dissolveur;

- un confinement dynamique pour accostage de châteaux de transport de combustibles; de fission;

- les conséquences d'un arrêt de refroidissement des stockages de produits retraitement.

- des transferts de contamination dans les gaz de procédé issus du 
Les techniques de simulation mises en œuvre sont de deux types : traçage à l'hélium et simulation par aérosols. Les applications de ces techniques sont nullement limitées aux usines de retraitement des combustibles irradiés. Le traçage à l'hélium en particulier est souvent utilisé pour résoudre des problèmes de ventilation dans des réseaux compliqués et de grande dimension, par exemple pour mettre en évidence des fuites et des by-pass dans les circuits de ventilation de centrales électriques.

\section{TRAÇAGE A L'HÉLIUM}

\section{a) Description de la technique}

De l'hélium est injecté dans un milieu gazeux (l'injection dans un milieu diphasique ou liquide sera envisagée plus loin). La mesure de la concentration en hélium en divers points de l'espace étudié est utilisée pour caractériser les conditions de transfert des points sources aux points de mesure.

L'appareillage mis au point pour ces interventions est un spectrographe de masse à l'hélium, associé à des dispositifs de prélèvement et d'étalonnage. Il offre les avantages suivants :

- mesure en temps réel et lecture directe de débits de gaz pouvant aller de $10^{-1}$ à $10^{6} \mathrm{~m}^{3} \cdot \mathrm{h}^{-1}$;

- précision : supérieure à $5 \%$;

- temps de réponse : de l'ordre de 1 seconde;

- absence absolue de toxicité.

Il peut être utilisé pour les gaz chauds ou corrosifs, quelle que soit la pression de la canalisation, en écoulement stationnaire ou rapidement variable.

\section{b) Fidélité du TRAÇAGE a L'HÉlium}

Avant d'examiner sur le plan théorique et pratique toutes les possibilités de traçage à l'hélium, il importe de dire d'abord que l'hélium trace fidèlement les mouvements convectifs des écoulements gazeux. Ce n'est pas, contrairement à une opinion répandue, un gaz qui diffuse tous azimuts dès son émission. En fait, le coefficient élevé de diffusion de l'hélium ne joue que sur quelques pour mille des molécules injectées, les autres restant portées par les mouvements convectifs. Les calculs suivants montrent cette fidélité du traçage à l'hélium.

Flux d'hélium dû à un gradient de concentration en hélium

Soit $R_{\mathrm{He}}$ le rayon du grand cercle d'interaction de l'atome d'hélium. Volume balayé par celui-ci en 1 seconde :

$$
\pi R_{\mathrm{He}}^{2} v_{\mathrm{He}} \quad\left[v, \text { vitesse }\left(\mathrm{m} \cdot \mathrm{s}^{-1}\right)\right] .
$$

Si l'unité de volume $\left(\mathrm{m}^{3}\right)$ contient $n$ molécules d'air (en négligeant les interactions He-He en se plaçant dans les conditions $n_{\mathrm{He}} / n_{\mathrm{air}}<10^{-3}$ ), le nombre de chocs par seconde devient :

$$
v=\pi R_{\mathrm{He}}^{2} \cdot v_{\mathrm{He}} \cdot n_{\mathrm{air}}\left(\mathrm{s}^{-1}\right) .
$$

vol. $16-\mathrm{N}^{\circ} 2$ 


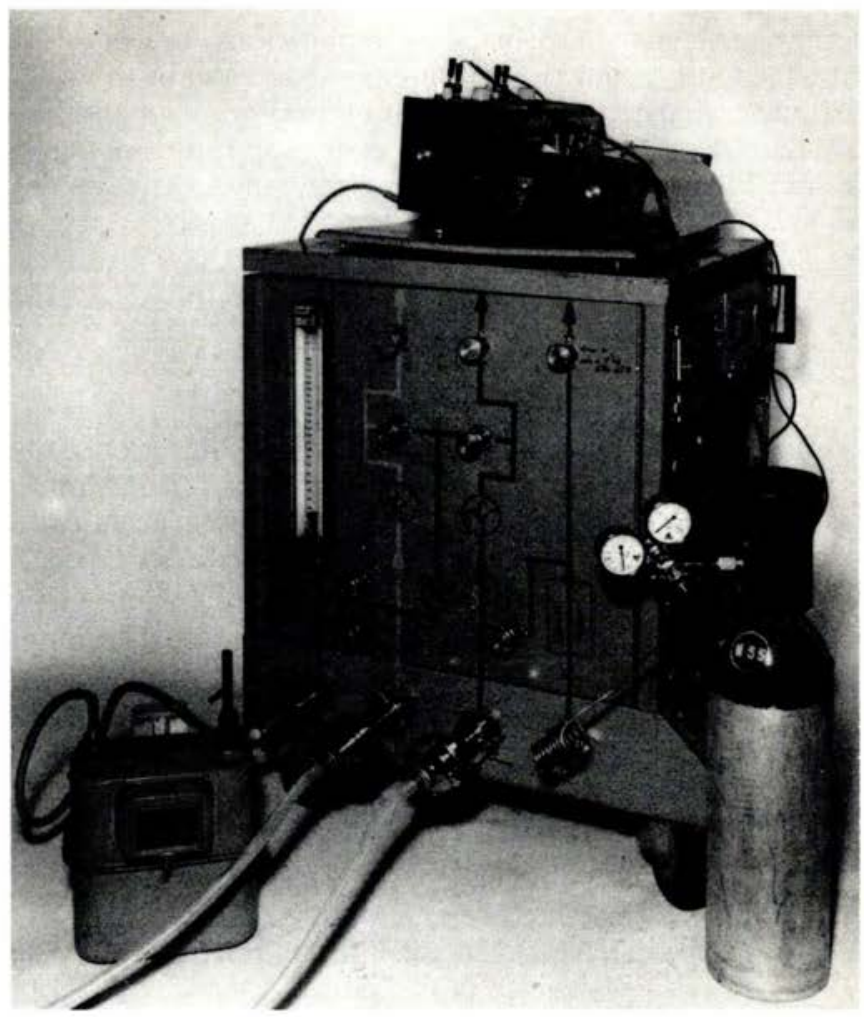

FIG. 1. - Débitmètre à hélium. Vue latérale du vannage de l'introduction des gaz.

Distance parcourue entre deux chocs (libre parcours moyen) $\lambda$ :

$$
\lambda=\frac{v_{\mathrm{He}}}{v}=\frac{1}{\pi R_{\mathrm{He}}^{2} n_{\mathrm{air}}} .
$$

Si de part et d'autre d'une surface, il existe un gradient $d n_{\mathrm{He}} / d x$, on montre qu'il apparaît un débit de fluence moyen $\delta$ de molécules d'hélium :

$$
\delta=0,50 \bar{v} \lambda \frac{d n_{\mathrm{He}}}{d x} \quad\left(\text { molécules } \cdot \mathrm{m}^{-2} \cdot \mathrm{s}^{-1}\right) \text {. }
$$

$\bar{v}$ est la vitesse moyenne tirée de l'intégration de l'équation de Boltzmann, soit :

$$
\bar{v}=\sqrt{\frac{8 k T}{\pi m}} .
$$


Application numérique:

$$
\begin{gathered}
R_{\mathrm{He}}=2,30 \cdot 10^{-10} \mathrm{~m}, \\
\bar{v}_{\mathrm{He}}=1250 \mathrm{~m} \cdot \mathrm{s}^{-1} \text { à } 20^{\circ} \mathrm{C}, \\
n_{\text {air }}=6 \cdot 10^{23} \cdot \frac{273}{293} \cdot \frac{10^{3}}{22,4}=2,5 \cdot 10^{25} .
\end{gathered}
$$

Si on se place dans le cas d'un gradient établi entre une source (air contenant $1000 \mathrm{ppm}$ d'hélium) et un puits pour l'hélium (par exemple le balayage d'air frais) situés à une distance $x$ (en mètres) :

$$
\frac{d n_{\mathrm{He}}}{d x}=\frac{2,5 \cdot 10^{22}}{x} .
$$

Le débit de fluence moyen des molécules d'hélium est :

$$
\delta=0,50 \cdot 1250 \cdot \frac{1}{\pi\left(2,3 \cdot 10^{-10}\right)^{2} \cdot 2,5 \cdot 10^{25}} \cdot \frac{2,5 \cdot 10^{22}}{x}=\frac{3,8 \cdot 10^{18}}{x},
$$

pour un gradient de $1000 \mathrm{ppm}$ :

$$
\delta \approx \frac{4 \cdot 10^{18}}{x} \text { molécules He } \cdot \mathrm{m}^{-2} \cdot \mathrm{s}^{-1} .
$$

$\mathrm{Si}$ on représente ce flux sous la forme d'une progression d'un front de $1000 \mathrm{ppm}$ vers un espace sans hélium, on obtient, pour un gradient de $1000 \mathrm{ppm} \cdot \mathrm{m}^{-1}$, la vitesse équivalente suivante :

$$
V=\frac{\delta}{n_{\mathrm{He}}}=\frac{4 \cdot 10^{18}}{2,5 \cdot 10^{22}}=1,6 \cdot 10^{-4} \cdot \mathrm{m} \cdot \mathrm{s}^{-1} .
$$

On voit donc que la vitesse moyenne de diffusion de l'hélium ne devient importante que lorsque les gradients de concentration sont très élevés : $V$ n'est supérieure au centimètre par seconde que si $x$ est inférieur au centimètre.

Les mouvements convectifs mettant en jeu des vitesses supérieures au centimètre par seconde, la représentativité du traçage des mouvements convectifs par l'hélium est donc assurée au centimètre près.

On pourrait craindre que la prise en compte seulement de $\bar{v}$ ne tienne pas compte des molécules rapides qui arriveraient plus rapidement. Si on intègre l'équation de Boltzmann par tranche de multiple de la vitesse moyenne :

$$
\bar{v}_{0}=\sqrt{\frac{8 k T}{\pi m}},
$$


on trouve :

- entre $(1 / 2) \bar{v}_{0}$ et $2 \bar{v}_{0} \simeq \%$ des molécules;

- entre $2 \bar{v}_{0}$ et $\infty \simeq 6 \%$ des molécules;

- entre $4 \bar{v}_{0}$ et $\infty<10^{-8}$ des molécules.

Le nombre de molécules de $\bar{v}>2 \bar{v}_{0}$ est donc tout à fait négligeable.

\section{c) Évaluation théoriQue et PRATIQUe du TRAÇAGE a L'HÉlium}

La méthodologie d'étude par traceur a été bien décrite par MM. GuIZERIX et Margrita dans le numéro spécial 1976 de La Houille Blanche où est développée la méthode des fonctions de transfert. Cette méthodologie, décrite dans le cas de l'eau, s'applique également à l'hélium dans l'air puisqu'il a été montré ci-dessus que l'hélium traçait fidèlement les mouvements convectifs.

Nous allons appliquer cette théorie de traçage au cas d'une enceinte ventilée où peut être générée une contamination (point $S$ ) pour laquelle nous allons examiner le profit que l'on peut tirer de l'injection d'hélium au point $S$ ou dans d'autres endroits de l'enceinte ou des conduits de ventilation.

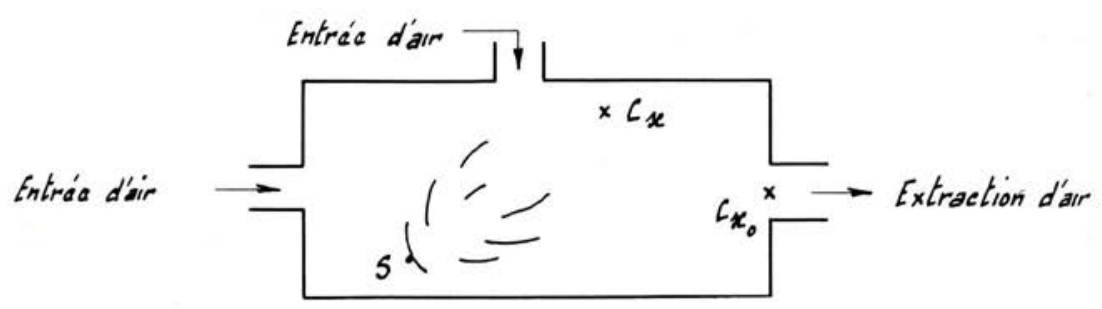

FIG. 2. - Schéma de l'enceinte ventilée. $x$, point quelconque de concentration $C_{x}$; $x_{0}$, point situé à l'aspiration de la gaine d'extraction, concentration $C_{x_{0}}$.

Génération d'hélium en $S$

- Injection instantanée d'une masse $m$ d'hélium

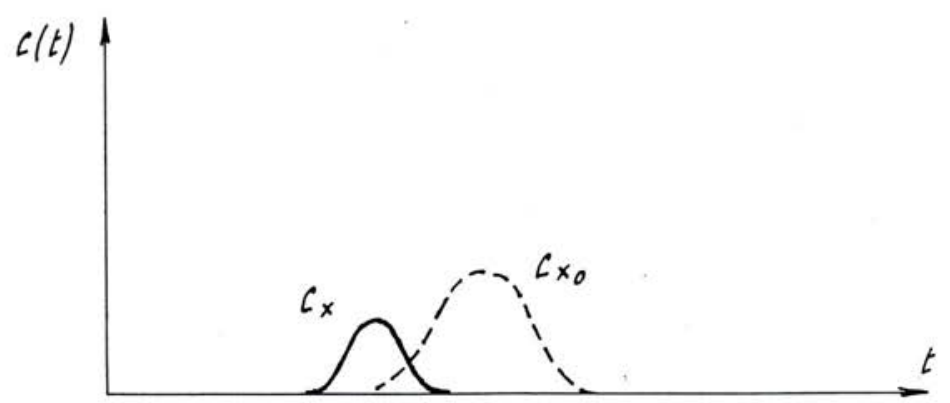

FIG. 3. - Injection instantanée d'une masse $m$ d'hélium. 
On obtient le temps de transit entre un point quelconque $x$ et un point $x_{0}$ situé à l'aspiration de la gaine d'extraction et la distribution des temps de séjour d une contamination ponctuelle aux points de mesure par les enregistrements des spectres de concentration schématisés ci-dessus.

La proportion de la contamination parvenue aux points $x$ et $x_{0}$ est respectivement :

$$
\frac{\int_{-\infty}^{+\infty} q C_{x}(t) d t}{m} \text { et } \frac{\int_{-\infty}^{+\infty} q_{0} C_{x_{0}}(t) d t}{m},
$$

$q$ étant le débit massique de gaz prélevé et $m$ la masse totale générée.

- Injection à débit constant d'hélium

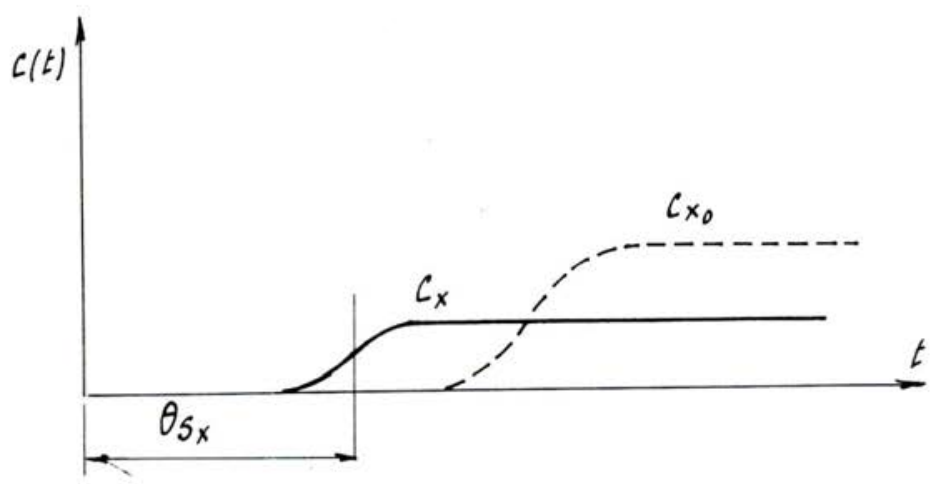

FıG. 4. - Injection à débit constant d'hélium.

En régime établi, la concentration en hélium présente un plateau. Le niveau de concentration atteint dépend du débit de gaz en $S$ et de la répartition de ces gaz vers les points de mesure.

La mesure de $\theta_{S x}$, date moyenne d'arrivée des premières bouffées d'hélium en $x$ et de $l_{S x}$, distance $S x$, fournit la vitesse moyenne de transit des gaz de $S$ vers $x$.

$$
\bar{v}_{S x}=\frac{l_{S x}}{\theta_{S x}} .
$$

Si l'on note plusieurs paliers, cela signifie qu'il y a des cellules de convection.

La mesure de la montée en concentration jusqu'au palier donne la constante de temps de renouvellement locale due au flux d'air transitant en $x$.

La mesure de la concentration en hélium en $x$, lorsqu'on la rapporte à la concentration en hélium générée en $S$, donne le facteur de dilution d'une contamination issue de $S$.

voL. $16-\mathrm{N}^{\circ} 2$ 
De plus, $C_{x} Q_{x}$ (débit local) est le flux d'hélium transitant en $x$. En supposant la conservation de la masse du traceur émis, $Q_{x_{0}} C_{x_{0}}=Q C$ $(Q$ : débit d'hélium généré à concentration $C$ ).

$Q_{x}$ est proportionnel à $Q_{x_{0}}$ dans le rapport des temps de montée en concentration en hélium (constantes de temps de renouvellement $\tau$ ) :

$$
Q_{x}=Q_{x_{0}} \frac{\tau_{x_{0}}}{\tau_{x}} .
$$

Finalement, le flux d'hélium transitant en $x$ est :

$$
C_{x} \cdot \frac{\tau_{x_{0}}}{\tau_{x}} \cdot \frac{Q C}{C_{x_{0}}}
$$

et la part de contamination transitant en $x$ est :

$$
\frac{C_{x}}{C_{x_{0}}} \cdot \frac{\tau_{x_{0}}}{\tau_{x}} .
$$

En pratique, ces calculs ne peuvent fournir que des ordres de grandeur des transferts de contamination car, surtout lorsque les vitesses d'air sont faibles, les régimes établis sont peu stables.

On se fixe donc des seuils de concentration par rapport à la concentration dans le domaine d'extraction, seuils au-dessous desquels on déclare qu'il y a peu de transfert de contamination.

d) Avenir de la technique

Cette technique de traçage rend d'énormes services pour pister semiquantitativement les transferts de contamination et pour mesurer certains paramètres essentiels de la ventilation tels que les débits.

A l'avenir, elle est appelée à connaître encore plus de succès par l'introduction d'un progrès technique : le prélèvement sélectif d'hélium par une membrane semi-perméable. Ceci permettra :

- d'accroître la stabilité de la mesure dans les gaz;

- d'opérer en milieu corrosif et radioactif car seul le point de prélèvement de la membrane et non le spectromètre subit l'agressivité du milieu;

- de mesurer l'hélium en milieu liquide. Cette dernière application ouvre un champ d'études très élargi et extrêmement intéressant quant au traçage de contaminants générés dans des liquides ou des milieux diphasiques.

\section{SIMULATION PAR AÉROSOLS}

a) Préliminaire

Un deuxième groupe de techniques consiste à simuler les transferts de contamination par aérosols.

Il est important pour la suite de cet article d'opérer dès à présent une distinction selon le niveau de connaissance du système où se produisent les transferts de contamination. Schématiquement, deux cas sont possibles : 
$1^{\text {er }}$ cas

La source de contamination est définie. Les principes physiques régissant les transferts sont bien connus. C'est le cas par exemple de la plupart des appareillages utilisés en filtration. On étudie alors par aérosols tests la fonction de transfert du système (de l'appareillage).

Les études de ce type sont souvent normalisées selon les dispositifs à tester [essais de filtre à l'uranine, au DOP $\left({ }^{1}\right)$...]. Nous avons mis au point pour notre part une procédure d'essai à l'uranine de matériel de préfiltration que nous avons utilisée pour améliorer l'efficacité des dévésiculeurs utilisés au C.E.A.

$2^{\mathrm{e}}$ cas

Les principes physiques de la génération et des transferts à l'intérieur du système sont mal connus. L'étude des phénomènes est délicate mais intéressante à approfondir pour définir les apports et limites de la simulation par aérosols. Dans ce qui suit, nous ne nous intéresserons plus qu'à ce cas, qu'on appelera système « flou ».

\section{b) DESCRIPTION DES MÉTHOdES UTILISÉES}

Dans l'étude d'un système " flou ", deux problèmes se posent :

- caractériser la source : physiquement (distribution, concentration ..., évolution physico-chimique) et géographiquement (lieu, direction d'émission);

- connaître le comportement des aérosols : rétention, réentraînement, et surtout, pour les vésicules, influence de la température, pression, milieu réactionnel.

Compte tenu de ce qu'on sait du système, il faut dès le départ répondre à la question suivante :

A-t-on avantage à séparer l'étude des problèmes de génération de ceux du transfert ou vaut-il mieux étudier globalement la réponse du système?

La solution est au cas par cas et dépend d'une évaluation a priori (jeu des lois physiques, existence de données expérimentales ...) de la séparabilité de ces deux problèmes.

Une fois cette question résolue (on peut contrôler parfois a posterior l'opportunité de la solution adoptée), on cherchera à simuler au mieux les transferts de contamination par le choix de l'aérosol.

Une distinction doit être opérée selon que la génération s'effectue en milieu gazeux ou en milieu liquide.

\section{Génération en milieu gazeux}

Les techniques que nous utilisons le plus souvent sont celles au sulfure de zinc $(\mathrm{ZnS})$ et à l'uranine. La technique au $\mathrm{ZnS}$ permet de mesurer des masses surfaciques extrêmement faibles grâce aux propriétés de scintillation du $\mathrm{ZnS}$ et à

( $\left.{ }^{1}\right)$ DOP, dioctyle phtalate.

vOL. $16-\mathrm{N}^{\circ} 2$ 
sa soúplessè d'utilisation. De plus, la génération de l'aérosol ne perturbe pas le milieu gazeux contrairement à ce qui se passe avec les fumigènes par exemple.

Le mode opératoire est le suivant :

La simulation de l'empoussièrement est réalisée grâce à une poudre de sulfure de zinc $(\mathrm{ZnS})$ de densité voisine de 2 et de granulométrie variable (selon les besoins, de 1 à $10 \mu \mathrm{m}$ ). Cette poudre de $\mathrm{ZnS}$ permet, grâce à un procédé original breveté par le S.T.E.P./S.P.T., de déterminer la masse de $\mathrm{ZnS}$ déposée sur un filtre plan (membrane inerte d'ester de cellulose ou de téflon) ou directement sur une paroi plane, avec une bonne précision et pour la gamme 0,1 $500 \mu \mathrm{g} . \mathrm{cm}^{-2}$. Le principe en est le suivant : un comptage de photons émis après bombardement par une source $\alpha$ est rendu possible du fait des propriétés luminescentes de $\mathrm{ZnS}$. L'appareillage développé par le S.T.E.P./S.P.T. comprend un photomultiplicateur auquel est associée une source $\alpha$ transparente et un tiroir de positionnement du filtre (dans le cas de la sonde fixe). Ce photomultiplicateur est relié à un appareil de comptage qui donne la valeur du comptage en chocs par seconde, et qui permet, après déduction du mouvement propre du compteur, de déterminer la masse de poudre de $\mathrm{ZnS}$ déposée par unité de surface grâce à une courbe d'étalonnage défınie par rapport à un dépôt étalon.

En ce qui concerne la technique de l'uranine, on génère des goutelettes de solution d'uranine de concentration connue et, par séchage, on aboutit à un résidu sec. En faisant varier la taille des gouttelettes et la concentration de la solution, on peut donc générer un aérosol monodispersé de la dimension voulue. Le domaine d'application déjà utilisé va de 0,6 à $15 \mu \mathrm{m}$, avec un aérosol dont la concentration numérique est d'environ 100 par centimètre cube.

\section{Génération en milieu liquide}

La production par arrachement au bouillonnement de vésicules peut amener un transfert de contamination. Pour simuler ce processus on peut remplacer le contaminant par un traceur soluble dans le liquide source. Une des qualités principales que doit posséder ce traceur est que son seuil de détection soit très faible de façon à pouvoir détecter des masses les plus petites possibles.

La solution que nous utilisons le plus souvent est une solution de sel de fluorescéine de concentration donnée.

On peut utiliser d'autres sels, tel que le chlorure de sodium, et on envisage même, pour certaines applications, de tracer l'eau par les impuretés solubles qu'elle contient (en supposant que les impuretés insolubles sont faibles par rapport aux impuretés solubles).

Parfois, on peut analyser directement les contaminants contenus dans les aérosols, comme cela a été le cas pour une étude des transferts de contamination entre l'eau d'une piscine de stockage et l'air ambiant du hall.

D'autre fois, on remplace les produits actifs par des produits inactifs de mêmes propriétés chimiques ou de propriétés chimiques très voisines. C'est ce que nous avons fait lors de la simulation d'une solution de produits de fission. 
Dans ces conditions, l'analyse pour caractériser la source (débit massique, distribution en dimension) peut s'envisager de deux façons :

- soit en effectuant des mesures directement sur les vésicules liquides;

- soit en effectuant des mesures sur le résidu sec de ces vésicules.

Dans le premier cas, la diffıculté de maintenir les vésicules en équilibre avec le fluide porteur peut amener soit un grossissement par condensation, soit une évaporation, ces deux processus étant la plupart du temps incontrôlés.

Dans le deuxièmecas,on procède au moment de l'analyse à une évaporation complète contrôlée ce qui évite toute modification en dimension intempestive. La diffıculté dans ce cas est de remonter à la taille originelle des vésicules. Néanmoins, cette conversion est facilement effectuée si l'on suppose que la concentration du traceur est identique dans le liquide source et dans les vésicules.

On obtient alors :

$$
\frac{D_{v}}{D_{r}}=\left(\frac{\rho_{r}}{C}\right)^{1 / 3},
$$

avec $D_{v}$ et $D_{r}$ diamètres respectifs des vésicules et du réseau sec $\rho_{r}$ masse volumique du résidu sec (masse volumique du traceur) et $C$ concentration du traceur dans la solution.

\section{c) ThÉorie de La SIMILITUde EN MÉCANIQUe DES AÉROSOlS}

La théorie de la similitude doit être utilisée lorsqu'on veut réduire la dimension des expériences consacrées au comportement des aérosols ou lorsqu'on ne peut reproduire exactement les caractéristiques de l'aérosol réel (granulométrie, densité).

Résumons ci-dessous les principaux résultats de cette théorie :

L'aérosol peut être régi par des forces d'inertie, de diffusion ou différents champs de forces extérieures (pesanteur, action électrostatique, thermophorèse ...).
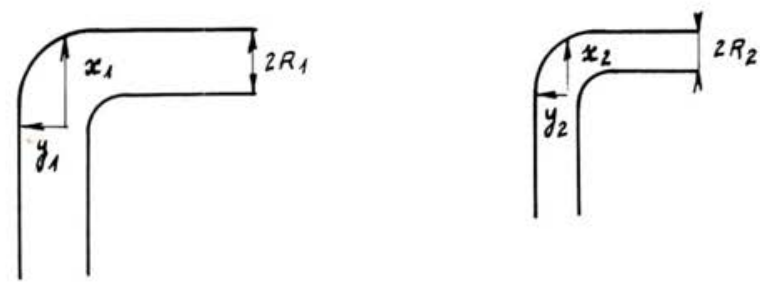

FIG. 5. - Similitude géométrique des conduits.

Pour que le déplacement de deux aérosols soit similaire, il est nécessaire d'avoir :

$1^{\circ}$ la similitude géométrique des canalisations;

$2^{\circ}$ la similitude de l'écoulement du fluide dans chaque système;

$3^{\circ}$ la similitude des trajectoires des particules. 
1) La similitude géométrique des conduits est exprimée par l'équation :

$$
\frac{x_{1}}{x_{2}}=\frac{y_{1}}{y_{2}}=\frac{R_{1}}{R_{2}}=\mathrm{Cte},
$$

où $x_{1}$ et $y_{1}$ sont les coordonnées d'un point quelconque dans le système $1, x_{2}$ et $y_{2}$ étant les coordonnées du point correspondant dans le système $2, R_{1}$ et $R_{2}$ étant des quantités caractérisant les dimensions linéaires des deux systèmes telles que le rayon d'un conduit.

2) La condition dynamique pour la similitude des écoulements des fluides dans deux systèmes géométriquement similaires est la conservation du nombre de Reynolds :

$$
\frac{2 U_{1} R_{1}}{v_{1}}=\frac{2 U_{2} R_{2}}{v_{2}}=\mathrm{Re}=\mathrm{Cte},
$$

$U_{1}$ étant la vitesse moyenne des écoulements dans le système $1, R_{1}$ le rayon du conduit et $v_{1}$ la viscosité cinématique du fluide; $U_{2}, R_{2}$ et $v_{2}$ sont les paramètres correspondants dans le système 2 .

3) La similitude du parcours des particules dans les deux systèmes est assurée par la conservation du nombre de Stokes :

$$
\frac{\tau_{1} U_{1}}{R_{1}}=\frac{\tau_{2} U_{2}}{R_{2}}=\mathrm{St}=\mathrm{Cte} .
$$

$\tau_{1}$ et $\tau_{2}$ représentent le temps de relaxation des particules dans les systèmes 1 et 2 . De façon générale $\tau$ a pour expression :

$$
\tau=\rho_{p} \frac{C \cdot D_{p}^{2}}{18 \mu},
$$

où $\rho_{p}$ représente la masse volumique de la particule, $C$ le facteur correctif de Cunningham, $D_{p}$ le diamètre de la particule et $\mu$ la viscosité dynamique du fluide.

Si les deux systèmes sont soumis à l'action de forces extérieures $F$ identiques conférant aux particules une accélération $a=F / m$ semblable, on obtient une autre condition de similitude :

$$
\frac{U_{1}^{2}}{2 R_{1} a}=\frac{U_{2}^{2}}{2 R_{2} a}=\text { Cte. }
$$

Dans le cas particulier des forces de gravité soit $a=g$, ce nombre sans dimension s'appelle le nombre de Froude (Fr) :

$$
\frac{U_{1}^{2}}{2 R_{1} g}=\frac{U_{2}^{2}}{2 R_{2} g}=\mathrm{Fr}=\text { Cte. }
$$

Ainsi la condition de similitude des trajectoires des particules dans des systèmes différents est l'égalité des nombres de Reynolds et de Stokes, l'égalité du nombre de Froude devant être assurée lorsque les forces de gravité sont considérées. 
La conservation simultanée de ces trois nombres d'un système à l'autre est rarement possible.

Aussi la combinaison de ces nombres peut amener à une présentation simplifiée des conditions. On peut obtenir ainsi :

$$
\frac{\mathrm{St}}{2 \mathrm{Fr}}=\frac{\tau U}{R} \cdot \frac{2 R g}{2 U^{2}}=\frac{\tau g}{U}=\frac{V_{s}}{U}=\mathrm{Cte},
$$

où $V_{s}$ représente la vitesse de sédimentation de la particule.

Si l'inertie des particules peut être négligée (cas de l'écoulement laminaire dans un conduit rectiligne) seule la condition précédente est à remplir, montrant ainsi que la trajectoire des particules dépend uniquement du rapport entre la vitesse de sédimentation des particules et la vitesse du fluide.

Au contraire,si l'inertie des particules est le phénomène prédominant une combinaison du type :

$$
\frac{\mathrm{Re}}{9 \mathrm{St}}=\frac{R^{2} \cdot \rho}{\left(D_{p} / 2\right)^{2} \cdot \rho_{p}}=\mathrm{Cte},
$$

où $\rho$ désigne la masse volumique du fluide, se simplifie énormément si les particules et le fluide sont de même nature dans les deux systèmes. La condition pour obtenir des trajectoires de particules similaires est alors :

$$
D_{p} / R=\text { Cte. }
$$

En conclusion, la qualité de la simulation dépend du respect des différentes contraintes et des simplifications susceptibles d'être effectuées.

\section{d) Exemple d'application}

A titre d'exemple, nous décrirons brièvement une étude de simulation de transfert de contamination par les aérosols récemment accomplie par notre Service.

Il s'agissait de caractériser le transfert des produits de fission (P.F.) émis sous forme d'aérosols lors de l'ébullition accidentelle, au demeurant fort improbable, des solutions de stockage de ces P.F.

L'étude s'est effectuée sur une maquette contenant 501 de solution non active de composition chimique analogue à la solution active.

Dans cette simulation, on a déterminé les caractéristiques physiques des aérosols (concentration en masse, distribution en dimension) et la composition en éléments du résidu sec de l'aérosol émis. La simulation a porté essentiellement sur la conservation de la puissance transmise par unité de volume à la solution. On ne s'est donc pas situé dans un cas parfait de similitude en mécanique des aérosols, mais pratiquement on doit faire souvent des compromis de ce type. 
Description du dispositif expérimental (fig. 6)

L'installation se compose :

- d'une cuve qui nous permet d'amener à ébullition les solutions simulées de P.F. Sa forme se justifie par la volonté de se rapprocher le plus possible des conditions réelles (vitesse des fluides; rapport de dimensions entre la cuve et l'évent, hauteur entre le niveau du liquide et l'évent ). Le chauffage de la solution est assuré par un cordon chauffant entourant la cuve;

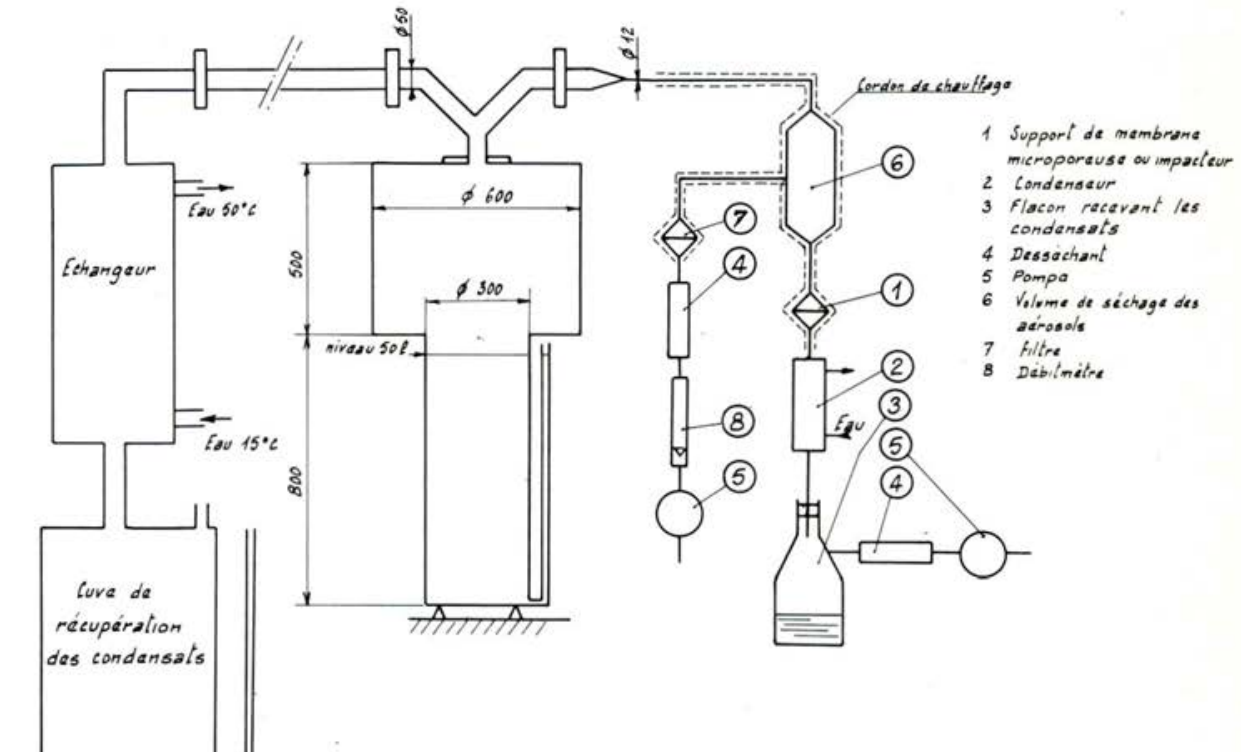

FiG. 6. - Dispositif expérimental de simulation de transfert de contamination.

- d'un échangeur à circulation d'eau qui est destiné à condenser les vapeurs émises lors de l'ébullition;

- d'une cuve qui nous permet de recueillir les condensats et de suivre ainsi les débits de vapeurs à l'ébullition;

- de différents dispositifs de collection des aérosols (filtres, membranes microporeuses, impacteur) ceux-ci servant à l'analyse a posteriori par pesée, microscopie optique ou électronique et par spectrographie.

La figure 6 représente la cuve, l'échangeur principal et la partie destinée à l'analyse des aérosols.

\section{Mesures}

Les mesures effectuées sont de deux types:

- débits des fluides : il s'agit essentiellement de déterminer les vitesses d'évaporation en mesurant le débit des condensats. 
- Caractéristiques des aérosols :

$1^{\circ}$ Caractéristiques physiques

- concentration massique par pesée gravimétrique,

- distribution en dimension par microscopie optique et électronique.

$2^{\circ}$ Caractéristiques chimiques

On détermine la nature des éléments rentrant dans la composition du résidu sec de l'aérosol par spectrographie, ce qui permet de la comparer avec la solution d'origine.

\section{Expériences}

On a choisi d'effectuer deux expériences identiques dans le but de tester la reproductibilité des résultats.

Une première expérience a été réalisée avec une évaporation par ébullition de $75 \%$ de la solution.

Une seconde expérience a été menée approximativement jusqu'à une évaporation de $85 \%$ de la solution.

Dans chaque expérience,de l'ordre de 20 prélèvements sur des membranes microporeuses ont été effectués au cours de l'ébullition. A la fin de l'ébullition un impacteur est utilisé pour les prélèvements, ce qui permet d'accéder directement à la distribution en masse de l'aérosol.

Cette étude nous a ainsi permis d'appréhender la variation du facteur de décontamination de la cuve au cours de l'ébullition et de caractériser la source de vésicules.

\section{CONCLUSION}

A l'aide de la gamme de traceurs qui vient d'être décrite (hélium, aérosols) on peut simuler la plupart des transferts de contamination et trouver, à coût modéré, des solutions aux problèmes que posent ces transferts de contamination.

Ces techniques de traçage permettent d'une part de simuler en inactif des phénomènes qu'il serait difficile ou cher d'étudier en milieu radioactif, d'autre part d'évaluer sur installations nucléaires en fonctionnement des transferts de contamination qu'il serait difficile, voire impossible, d'appréhender par les analyses radioactives ou physico-chimiques classiques.

Grâce à une meilleure évaluation des transferts de contamination, on peut améliorer la radioprotection et la sûreté radiologique d'une installation en conception ou en exploitation et en particulier, optimiser le dimensionnement des circuits de ventilation qui participent de façon privilégiée au confinement de l'installation. 